\title{
Article \\ Fisher and Skew Information Correlations of Two Coupled Trapped Ions: Intrinsic Decoherence and Lamb-Dicke Nonlinearity
}

\author{
Abdel-Baset A. Mohamed ${ }^{1,2}\left(\mathbb{D}\right.$, Ahmed Farouk $^{3}\left(\mathbb{D}\right.$, Mansour F. Yassen ${ }^{1, *(D)}$ and Hichem Eleuch ${ }^{4,5,6}$ (D) \\ 1 Department of Mathematics, College of Science and Humanities in Al-Aflaj, Prince Sattam Bin Abdulaziz \\ University, Alkharj 11942, Saudi Arabia; abdelbastm@aun.edu.eg \\ 2 Department of Mathematics, Faculty of Science, Assiut University, Assiut 71515, Egypt \\ 3 Department of Computer Science, Faculty of Computers and Artificial Intelligence, South Valley University, \\ Hurghada 84511, Egypt; ahmed.farouk@sci.svu.edu.eg \\ 4 Department of Applied Physics and Astronomy, University of Sharjah, Sharjah 27272, United Arab Emirates; \\ heleuch@sharjah.ac.ae \\ 5 Department of Applied Sciences and Mathematics, College of Arts and Sciences, Abu Dhabi University, \\ Abu Dhabi 59911, United Arab Emirates \\ 6 Institute for Quantum Science and Engineering, Texas A\&M University, College Station, TX 77843, USA \\ * Correspondence: mf.ali@psau.edu.sa
}

check for

updates

Citation: Mohamed, A.-B.A.; Farouk,

A.; Yassen, M.F.; Eleuch, H. Fisher and Skew Information Correlations of Two Coupled Trapped Ions: Intrinsic Decoherence and Lamb-Dicke Nonlinearity. Symmetry 2021, 13, 2243. https://doi.org/10.3390/sym 13122243

Academic Editors: Pedro D. Sacramento and Nikola Paunkovic

Received: 9 October 2021

Accepted: 9 November 2021

Published: 24 November 2021

Publisher's Note: MDPI stays neutral with regard to jurisdictional claims in published maps and institutional affiliations.

Copyright: (c) 2020 by the authors. Licensee MDPI, Basel, Switzerland. This article is an open access article distributed under the terms and conditions of the Creative Commons Attribution (CC BY) license (https:/ / creativecommons.org/licenses/by/ $4.0 /)$.

\begin{abstract}
It is well known that many quantum information processing methods in artificial atoms depend largely on their engineering properties and their ability to generate quantum correlations. In this paper, we investigate the non-classical correlation dynamics of two trapped ions by using local quantum Fisher information, local quantum uncertainty, as well as logarithmic negativity. The system engineering is designed such that the two-trapped-ions work as two diploe-coupled qubits in a Lamb-Dicke regime. The center-of-mass vibrational modes are initially in coherent/even coherent states. It is found that the two-trapped-ions correlations can be controlled by the LambDicke nonlinearity, the nonclassicality effect of the initial center-of-mass vibrational mode, as well as the trapped-ion coupling and the intrinsic decoherence. The sudden changes in the non-classical correlations and their stability are shown against Lamb-Dicke nonlinearity, the nonclassicality, the trapped-ion coupling, and the intrinsic decoherence.
\end{abstract}

Keywords: trapped ions; local quantum Fisher information; local quantum uncertainty; logarithmic negativity

\section{Introduction}

Generating multiqubit correlated states is an efficient tool in quantum information [1-4]. Entanglement represents a special type of non-classical correlations (NCCs) and occupies a privileged position among them. It has many potential applications in quantum cryptography, teleportation, and quantum metrology. There are different entanglement measures, which their definitions depend on the nature of quantum systems (closed/open system) as: von Neumann entropy [5] for closed systems, negativity [6] and concurrence [7] for the open systems, and others. Recently, another definition of entanglement (for a quantum system of particles related to their entanglement with each other and the environment) based on the overlap of wave functions of identical particles is proposed [8]. After introducing quantum discord [9] as another type of NCC beyond entanglement, several quantifiers were proposed and used to quantify other non-classical correlations [10-14]. These quantifiers were defined by using skew information quantity [15] (as local quantum uncertainty (LQU) [16]), quantum Fisher information [17] (as local quantum Fisher information (LQFI) [18]), and geometrical correlation measures [19].

Quantum Fisher information is a useful quantifier to estimate different information resources as the entanglement, quantum speedup [20], and quantum phase transition [21]. It also has practical applications in quantum metrology [22]. Based on the minimal quantum 
Fisher information [17], LQFI [23] was introduced to enhance the efficiency and precision of quantum metrology protocols [24]. We will use the LQU and LQFI to quantify the degenerated correlations. It is proven that the skew information is a specific case of the quantum Fisher information [25].

However, the exploitation of the non-classical correlations is limited by the engineering properties of natural and artificial atomic systems (qubit models), which were used to implement a different type of quantum computation [26-28]) and quantum simulation [29]. Artificial two-level atoms (qubits) of trapped ions [30] were used to design a material platform for realizing quantum information [31] due to its high degree of controllability and motional degrees of freedom [32]. Multi-qubit trapped ions models were proposed for solving substantial computational problems [33-35].

The dynamics of the trapped-ion qubits depend on the center-of-mass-motion vibrational modes [36,37], and they have been proposed to exhibit Jaynes-Cummings dynamics $[38,39]$. The nonlinear dynamics of trapped-ion systems can be realized beyond the Lamb-Dicke regime [40-42]. In the Lamb-Dicke (LD) regime, where the LD nonlinearity is very small, the trapped-ion qubits can be cooled down to their ground state [43], and non-classical effects can be generated [44,45].

The dipole-dipole interaction between two trapped-ion qubits can be realized and observed experimentally [46,47]. It is used to implement quantum gates [48]. Quantum coherence created from the superposition of quantum states is playing a major role in applications in quantum information [49,50]. It is a necessary condition for creating nonclassical correlations. Therefore, we consider a superposition of coherent states that has a strong nonclassicality as an initial state for the center-of-mass vibrational mode fields.

The NCC investigations are limited by the phenomenon of intrinsic decoherence (ID) that can be realized in trapped-ion systems [51]. It differs from what occurs when the trapped-ion system is coupled to its environment but constitutes a major obstacle when a closed quantum system evolves. The coherence is usually destroyed by such dynamics [52].

In this paper, we are interested in analyzing the dynamical behavior of log-negativity entanglement, the LQU, and the LQFI correlations in two dipole-dipole coupled trappedions beyond the Lamb-Dicke regime. We study the effects of the intrinsic decoherence and the nonclassicality effect of the initial center-of-mass vibrational mode by considering the vibrational mode initially in two different coherent states.

The paper is structured as follows: the two considered trapped ions physical model is presented in Section 2. The definitions of the log-negativity, the LQU, and the LQFI, as well as their dynamical behaviors, are presented in Sections 3 and 4. In Section 5, we conclude our investigation.

\section{The Trapped-Ion Physical Model}

Here, we consider two coupled two-level cold ions beyond the Lamb-Dicke regime; each cold-ion system can be designed as a qubit. In the resolved sideband limit and the resonant case (where the laser and the $k$-th vibrational sideband have the frequency) $[40,53]$, the two ion's Hamiltonians are given by

$$
\begin{aligned}
\hat{H}_{0} & =\omega_{A} \hat{a}_{A}^{\dagger} \hat{a}_{A}+\omega_{B} \hat{a}_{B}^{\dagger} \hat{a}_{B}+\omega_{0}\left(\hat{\sigma}_{z}^{A}+\hat{\sigma}_{z}^{B}\right) \\
& +\sum_{l=A, B} K_{l} E_{l} e^{\left(i \theta_{l}-\left|\mu_{l}\right|^{2}\right)} \sum_{m} \frac{\left(-i \mu_{j}\right)^{2 m+k}}{(m+k) ! m !} \hat{a}_{l}^{\dagger m} \hat{a}_{j}^{m}\left(\hat{a}_{l}^{k}\left|1_{j}\right\rangle\left\langle 0_{j}\left|+\hat{a}_{l}^{\dagger k}\right| 0_{j}\right\rangle\left\langle 1_{l}\right|\right),
\end{aligned}
$$

where $\theta_{l}$ represents the initial applied laser phase of $l$-trap. $\hat{\sigma}_{z}^{l}$ designs the atomic flip operator between the upper $|1\rangle_{l}$ and lower $|0\rangle_{l}(l=A, B)$ levels with the frequency $\omega_{0}, K_{l}$ is the transition dipole moment. $E_{l}$ represents the amplitude of the applied laser $l$-field. While $\hat{a}_{l}$ and $\hat{a}_{l}^{\dagger}$ are the lowering and raising operators of the center-of-mass vibrational modes with the frequency $\omega_{l}(l=A, B) \cdot \mu_{l}$ is the Lamb-Dicke parameter of the trapped atomic $l$-ion. 
Based on the procedure described for engineering $N$ two-level cold ions beyond the Lamb-Dicke limit $[35,54,55]$, we consider the case where the two-level cold ions (two-qubit system) are closed to each other, and the total center of motion of them is the center-of-mass vibration of the two cold ions. Therefore, the vibrations of each ion frequency $\omega_{l}(l=A, B)$ are replaced by the total center of motion frequency $\omega$. For the resonant case, we take $\omega_{l}=\omega_{0}=\omega, \mu_{l}=\mu$. Here, the proposed investigation is motivated by the fact that the dipole-dipole coupling cannot be neglected if the standard rotating-wave approximation is considered [46,47]. Consequently, the two-qubit Hamiltonian is

$$
\begin{aligned}
\hat{H}= & \omega \hat{a}^{\dagger} \hat{a}+\omega\left(\hat{\sigma}_{z}^{A}+\hat{\sigma}_{z}^{B}\right)+\lambda \sum_{i=A, B}\left(\left|0_{i}\right\rangle\left\langle 1_{i}\right| \hat{a}^{\dagger k} F_{\mu}\left(\hat{a}^{\dagger} \hat{a}\right)\right. \\
& +\left|1_{i}\right\rangle\left\langle 0_{i}\right| F_{\mu}^{*}\left(\hat{a}^{\dagger} \hat{a}\right) \hat{a}^{k}+D\left(\left|1_{A} 0_{B}\right\rangle\left\langle 0_{A} 1_{B}|+| 0_{A} 1_{B}\right\rangle\left\langle 1_{A} 0_{B}\right|\right),
\end{aligned}
$$

where $\lambda_{l}=K_{l} E_{l} e^{i \theta_{l}} e^{-|\mu|^{2}}=\lambda(l=A, B), D$ represents the dipole-dipole coupling. By using the associated Laguerre $(k, \mu)$-polynomials $L_{m}^{k}\left(\mu^{2}\right)(m=0,1,2, \ldots)$, the diagonal elements of the nonlinear function operator $F_{\mu}\left(\hat{a}^{\dagger} \hat{a}\right)[40]$ is given by

$$
F_{\mu}(m)=\frac{m !}{(m+k) !} L_{m}^{k}\left(\mu^{2}\right) .
$$

The dynamics of the two cold ions' interactions with the intrinsic decoherence are analytically explored by the Milburn model [52]. In terms of the system density matrix $\hat{M}(t)$, the Milburn equation is given by

$$
\frac{d \hat{M}(t)}{d t}=-i[\hat{H}, \hat{M}(t)]-\frac{\gamma}{2}[\hat{H},[\hat{H}, \hat{M}(t)]],
$$

where $\gamma$ is the intrinsic decoherence parameter.

To investigate the ability of the two cold-ions interactions to generate entangled twoqubit states, we find a particular analytical solution for Equation (4), when the two trapped ions are initially in a disentangled state, in particular the upper state $\left|1_{A} 1_{B}\right\rangle$. With this state, all the considered correlations must vanish. The nonclassicality effect of the initial centerof-mass vibrational mode on the two ion-qubit dynamics are examined by considering the vibrational mode initially in two different coherent states. One of them is the coherent state (CS) that is given by

$$
\left|C S_{\alpha}\right\rangle=e^{-N / 2} \sum_{m=1}^{\infty} \frac{N^{\frac{m}{2}}}{\sqrt{m !}}|m\rangle .
$$

The initial intensity coherent field is designed by $N=|\alpha|^{2}$. The other is the even coherent state (ECS), which is characterized by its high nonclassicality. It is given by

$$
\left|E C S_{\alpha}\right\rangle=\frac{e^{-N / 2}}{\sqrt{2+2 e^{-2 N}}} \sum_{m=1}^{\infty} \frac{\left[1+(-1)^{m}\right] N^{\frac{m}{2}}}{\sqrt{m !}}|m\rangle,
$$

Therefore, the initial density matrix of the two cold-ions $M(0)$ is given by

$$
M(0)=\sum_{m, n=1}^{\infty} P_{m n}\left|m, 1_{A} 1_{B}\right\rangle\left\langle n, 1_{A} 1_{B}\right|
$$

where $P_{m n}$ represents the photon number distribution of the coherent/even-coherent state.

Here, we use the eigenstates of the Hamiltonian Equation (2) to find an analytical solution of Equation (4). 
On the basis of the two-level cold ions: $\left\{\left|S_{1}^{m}\right\rangle=\left|1_{A} 1_{B}, m\right\rangle,\left|S_{2}^{m}\right\rangle=\mid 1_{A} 0_{B}, m+\right.$ $\left.k\rangle,\left|S_{3}^{m}\right\rangle=\left|0_{A} 1_{B}, m+k\right\rangle,\left|S_{4}^{m}\right\rangle=\left|0_{A} 0_{B}, m+2 k\right\rangle\right\}$ with $m=0,1,2, \ldots$, the eigenstates $\left|E_{i}\right\rangle$ are given by

$$
\left|U_{j}\right\rangle=\sum_{n=1}^{4} X_{j n}^{m}\left|S_{n}^{m}\right\rangle,(j=1-4)
$$

where $X_{j n}^{m}$ satisfies the eigenvalue-problem: $\hat{H}\left|U_{j}^{m}\right\rangle=E_{j}^{m}\left|U_{j}^{m}\right\rangle$ based on the eigenvalues

$$
\begin{aligned}
E_{1}^{m}= & \omega(m+k), \\
E_{2}^{m}= & \omega(m+k)-D, \\
E_{3}^{m}= & \omega(m+k)+\frac{1}{2} D \\
& \quad-\lambda\left[\frac{D^{2}}{\lambda^{2}}+\frac{2(m+k) ! F_{\mu}^{2}(m+k)}{m !}+\frac{2(m+2 k) ! F_{\mu}^{2}(m+2 k)}{(m+k) !}\right]^{0.5}, \\
E_{4}^{m}= & \omega(m+k)+\frac{1}{2} D \\
\quad & \quad+\lambda\left[\frac{D^{2}}{\lambda^{2}}+\frac{2(m+k) ! F_{\mu}^{2}(m+k)}{m !}+\frac{2(m+2 k) ! F_{\mu}^{2}(m+2 k)}{(m+k) !}\right]^{0.5},
\end{aligned}
$$

Using the initial state of Equation (7) and the eigenvalues $E_{j}^{m}(j=1-4)$ and the eigenstates $\left|U_{j}^{m}\right\rangle$ of the Hamiltonian Equation (2), the time-dependent two trapped-ions density matrix is given by

$$
\begin{aligned}
\hat{M}(t)= & \sum_{m, n=0} P_{m n}\left[Y_{11}\left|U_{1}^{m}\right\rangle\left\langle U_{1}^{n}\left|+Y_{31}\right| U_{3}^{m}\right\rangle\left\langle U_{1}^{n}\right|\right. \\
& +Y_{41}\left|U_{4}^{m}\right\rangle\left\langle U_{1}^{n}\left|+Y_{13}\right| U_{1}^{m}\right\rangle\left\langle U_{3}^{n}\left|+Y_{33}\right| U_{3}^{m}\right\rangle\left\langle U_{3}^{n}\right| \\
& \left.+Y_{43}\left|U_{4}^{m}\right\rangle\left\langle U_{3}^{n}\left|+Y_{14}\right| U_{1}^{m}\right\rangle\left\langle U_{4}^{n}\left|+Y_{34}\right| U_{3}^{m}\right\rangle\left\langle U_{4}^{n}\left|+Y_{44}\right| U_{4}^{m}\right\rangle\left\langle U_{4}^{n}\right|\right],
\end{aligned}
$$

$Y_{j k}$ is given by

$$
Y_{j k}=X_{j 1}^{m} X_{k 1}^{m} \Lambda_{m n}^{j k}(t) T_{m n}^{j k}(t),
$$
fined by

The unitary evolution $\Lambda_{m n}^{j k}(t)$ and the intrinsic decoherence $T_{m n}^{j k}(t)$ terms are de-

$$
\Lambda_{m n}^{j k}(t)=e^{-i\left(E_{j}^{m}-E_{k}^{n}\right) t}, \quad T_{m n}^{j k}=e^{-\frac{\gamma}{2}\left(E_{j}^{m}-E_{k}^{n}\right)^{2} t} .
$$

Our aim is to explore the dynamics of the NCCs between the two trapped ions. We then need to find the two-ion reduced density matrix by considering the trace of the vibrational mode states from the system density matrix $M(t)$. The time-dependent two-ion state is obtained by

$$
M^{A B}(t)=\operatorname{tr}_{F}\{M(t)\} .
$$

For the one-photon case $k=1$, we can investigate the parameter effects of the LambDicke nonlinearity, the vibrational mode nonclassicality, the trapped-ion coupling, and the decoherence on the NCCs between the two trapped ions.

\section{Correlation Quantifiers}

Here, we introduce the definitions of the used non-classical correlations quantifiers; the LQFI and the atomic local quantum uncertainty, as well as the logarithmic negativity. 


\subsection{Logarithmic Negativity (LGN)}

The logarithmic negativity was used to investigate trapped-ion entanglement [6] in different real systems $[56,57]$. We determine the logarithmic negativity between the two trapped-ion qubits by using the negative eigenvalues $\lambda_{k}$ of the partial transpose of the two trapped-ions density matrix $\hat{M}(t)$ of Equation (9) with respect to a trapped-ion subsystem. Its closed formula is given by

$$
N(t)=\log \left[1+2\left|\sum_{k} \lambda_{k}\right|\right]
$$

The logarithmic negativity vanishes for uncorrelated states.

\subsection{Local Quantum Fisher Information (LQFI)}

Recently, local quantum Fisher information was introduced as another important quantum correlation quantifier. For a given quantum state (say a two-atom system) $M^{A B}(t)=\sum_{m} \pi_{m}\left|\psi_{m}\right\rangle\left\langle\psi_{m}\right|$ with $\pi_{m} \geq 0$ and $\sum_{m} \pi_{m}=1$, the local quantum Fisher information is defined as $[18,23]$

$$
F(t)=1-\pi_{W}^{\max }
$$

Based on the Pauli matrices $\sigma^{i}(i=1,2,3)$ and the matrices $\hat{H}_{i}=I \otimes \sigma^{i}$, we can calculate the highest eigenvalue $\pi_{W}^{\max }$ of the symmetric matrix $W$ of the elements

$$
w_{i j}=\sum_{\pi_{m}+\pi_{n} \neq 0} \frac{2 \pi_{m} \pi_{n}}{\pi_{m}+\pi_{n}}\left\langle\psi_{m}\left|\hat{H}_{i}\right| \psi_{n}\right\rangle\left\langle\psi_{n}\left|\hat{H}_{j}^{\dagger}\right| \psi_{m}\right\rangle .
$$

\subsection{Local Quantum Uncertainty}

Based on the Wigner-Yanase skew information quantity [15], the local quantum uncertainty (LQU) [58] was introduced to quantify another type of the nonlocal correlations. For a quantum state, with two trapped-ions state $M^{A B}(t)$, and a local observable with an operator $\hat{K}$, the LQU is defined as [16]

$$
L\left(M^{A B}(t)\right)=\min _{\hat{K}} I\left(M^{A B}(t), \hat{K}\right),
$$

where $I\left(M^{A B}(t), \hat{K}\right)$ is the skew information quantity [15]. For the trapped-ion state system that is identified by $M^{A B}(t)$, the LQU reduces to [58]

$$
L(t)=1-\lambda_{\max }\left(W_{A B}\right),
$$

where $\lambda_{\max }$ is the largest eigenvalue of the $3 \times 3$-matrix $W_{A B}$ defined by the following matrix elements

$$
w_{i j}=\operatorname{Tr}\left\{\sqrt{M^{A B}(t)}\left(\sigma_{i} \otimes I\right) \sqrt{M^{A B}(t)}\left(\sigma_{j} \otimes I\right)\right\},
$$

and $\sigma_{i(j)}, i=1,2,3$ are the Pauli operators.

After introducing quantum discord [9] (which is a more general way to measure NCCs than entanglement $[59,60]$ ) as another type of NCC beyond entanglement, several quantifiers were presented to quantify other NCCs, such as LQFI, LQU, and LGN. Each quantifier of the LQFI, LQU, and LGN detects a different kind of correlation due to the fact that their definitions depend on different mathematical expressions. The LGN depends on the partial transpose of the two trapped-ions density matrix, the LQU based on the Wigner-Yanase skew information quantity, while the LQFI depends on the minimum quantum Fisher information. 


\section{Dynamics of Correlations}

The non-classical correlation dynamics, the LQFI, and the atomic local quantum uncertainty, as well as the logarithmic negativity, are shown in Figures 1-6 under the effects of the Lamb-Dicke (LD) nonlinearity, the initial center-of-mass vibrational mode nonclassicality, as well as the trapped-ion coupling (TIC) and the intrinsic decoherence.
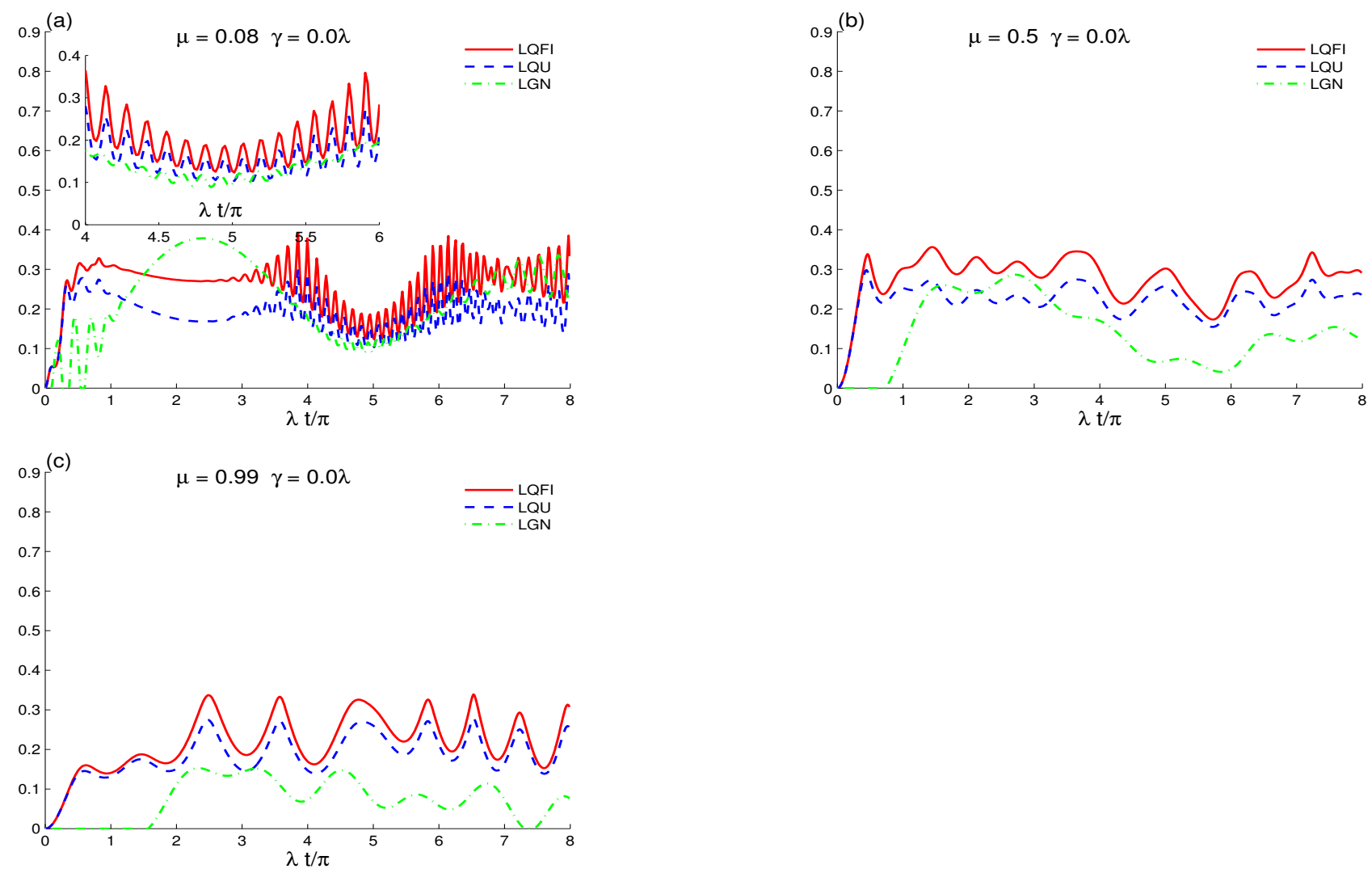

Figure 1. Time evolutions of the LQFI, LQU, and LGN non-classical correlations for $N=16$ with different Lamb-Dicke nonlinearity values: $\mu=0.08$ in (a), $\mu=0.5$ in (b), and $\mu=0.99$ in (c) in the absence of the intrinsic decoherence $\gamma=0$ and the dipole-dipole coupling $D=0$. When the vibrational mode state is initially in the coherent state.
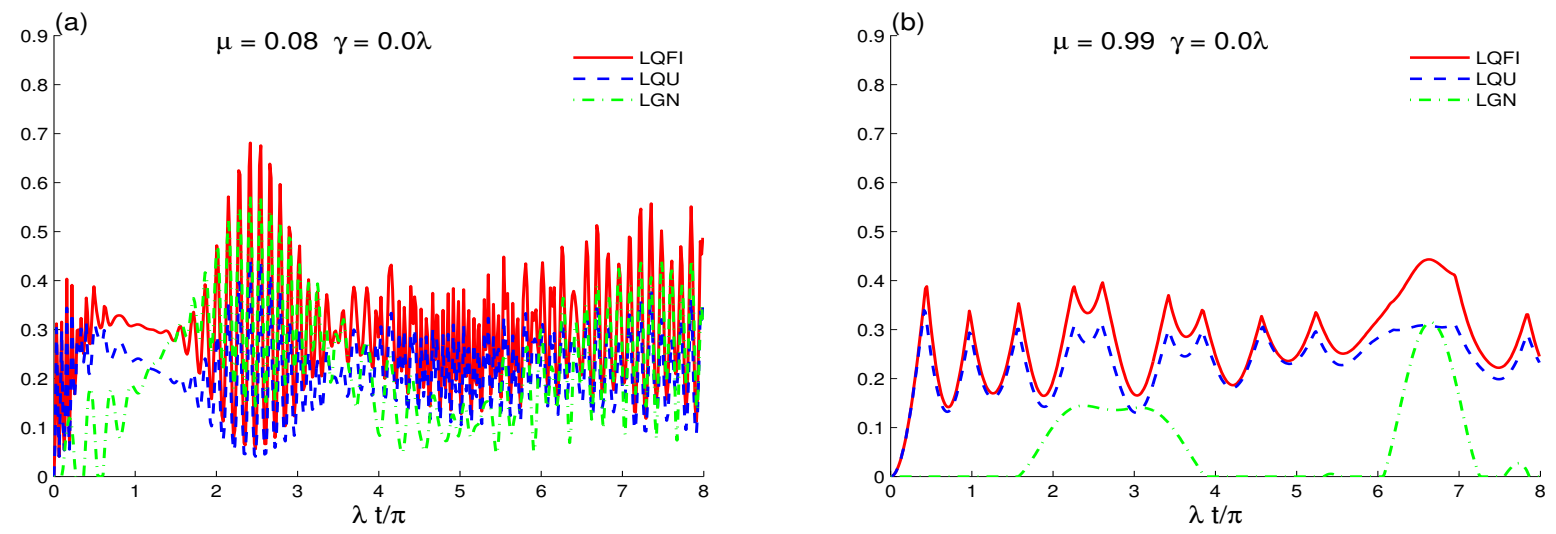

Figure 2. The dynamics of the LQFI, LQU, and LGN as in Figure 1a,c, but when the vibrational state is initially in the even superposition coherent state for different Lamb-Dicke nonlinearity values: $\mu=0.08$ in (a) and $\mu=0.99$ in (b). 

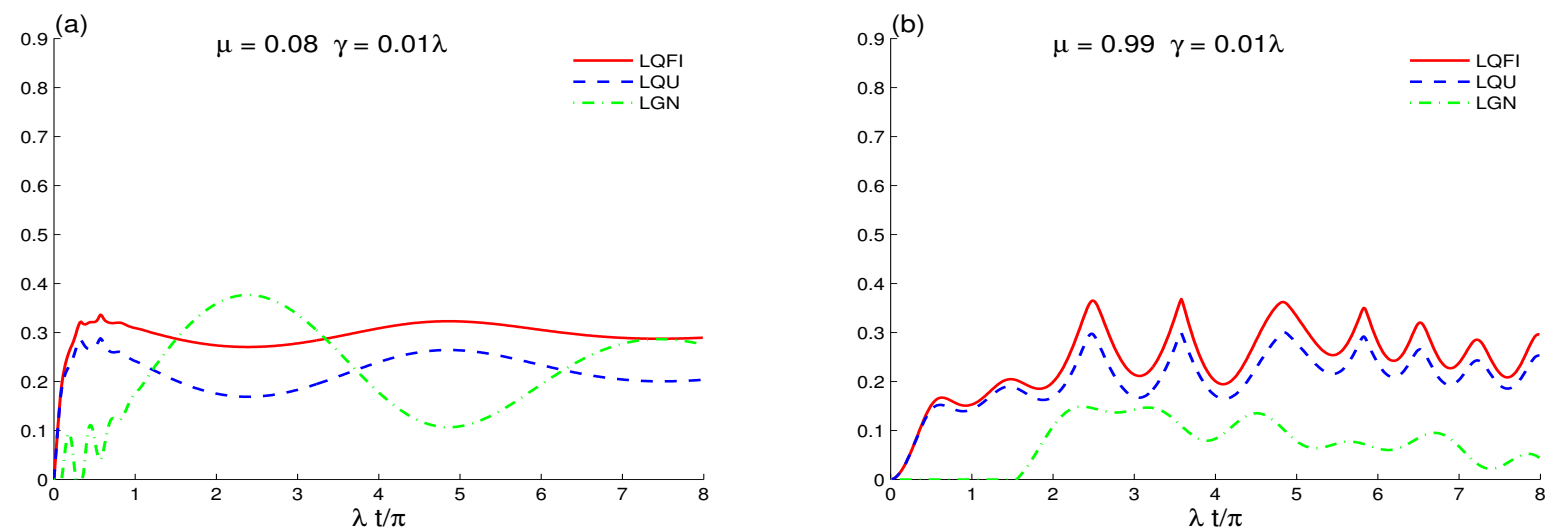

Figure 3. LQFI, LQU, and LGN dynamics as in Figure 1a,c, but in the presence of the intrinsic decoherence for different Lamb-Dicke nonlinearity values: $\mu=0.08$ in (a) and $\mu=0.99$ in (b).
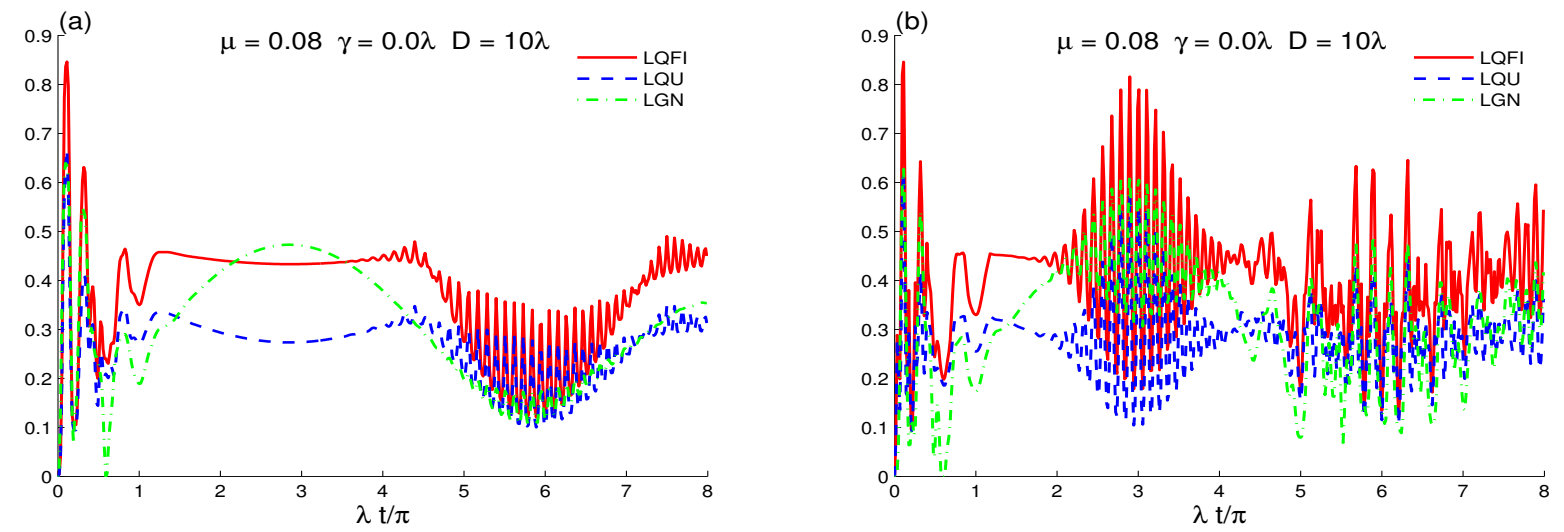

Figure 4. Non-classical correlation dynamics as in Figures 1a and 2a, but in the presence of the two trapped-ion couplings, $D=10 \lambda$. When the vibrational mode state is initially in the coherent state in (a) and the even coherent state in (b).
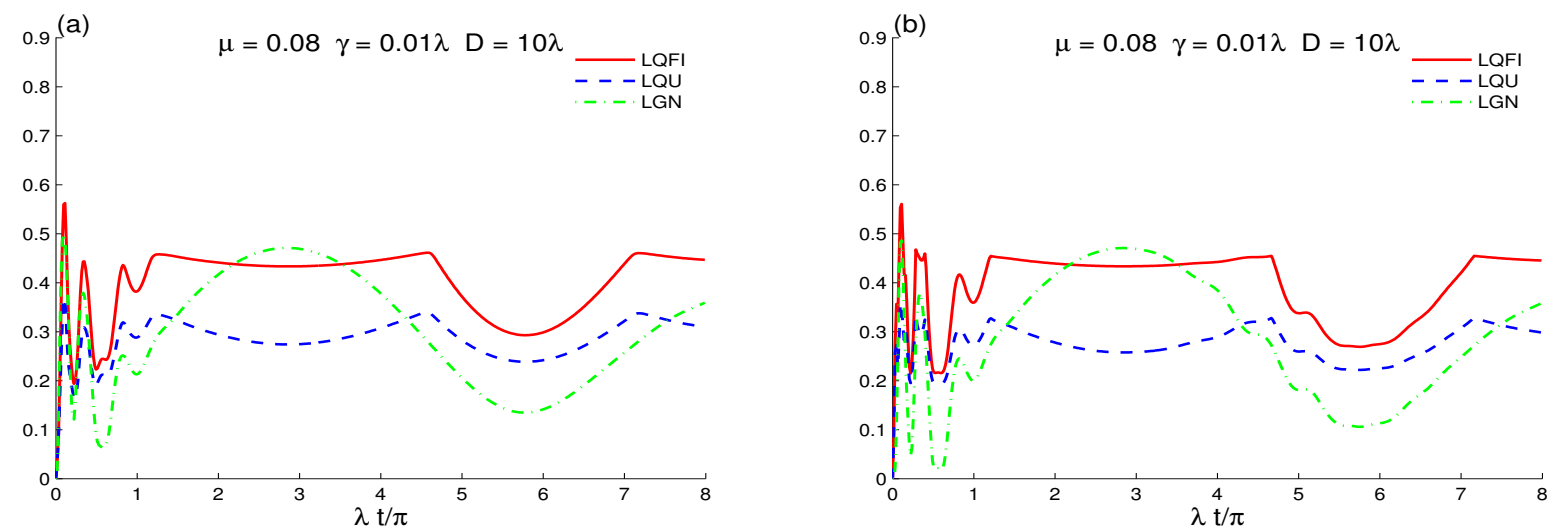

Figure 5. LQFI, LQU, and LGN correlation dynamics as in Figure 4, but for the two trapped-ion couplings $D=10 \lambda$ and the decoherence $\gamma=0.01 \lambda$. When the vibrational mode state is initially in the coherent state in (a) and the even coherent state in (b). 

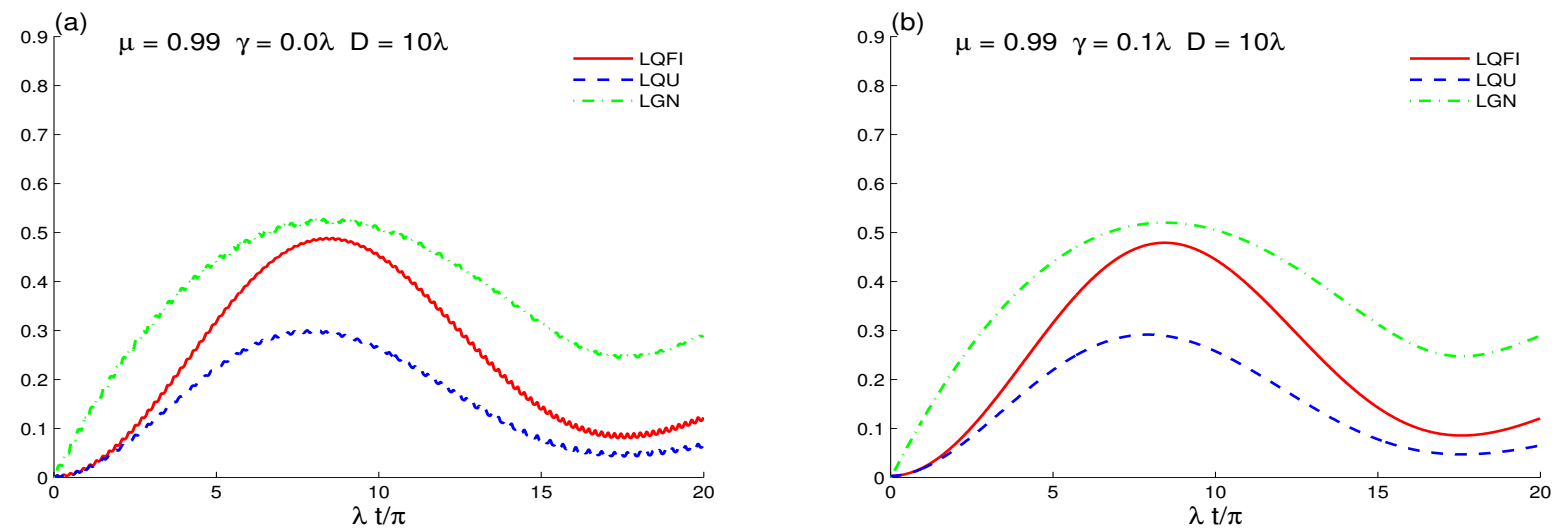

Figure 6. LQFI, LQU, and LGN dynamics, as in Figure 1c, but in the presence of the two trapped-ion couplings with a high Lamb-Dicke nonlinearity. When the vibrational mode state is initially in the coherent state for different intrinsic decoherence values: $\gamma=0.0 \lambda$ in (a) and $\gamma=0.1 \lambda$ in $(\mathbf{b})$.

Figures 1 and 2 show the amount of the generated non-classical correlations due to the unitary qubit-mode interactions. In this case, the intrinsic decoherence is absent, $\gamma=0$. From Equation (10), we find that the Rabi frequency $\Omega$ depends on the LambDicke nonlinearity $\mu$, the mean photon number $N$, and the trapped-ion coupling $D, \Omega \propto$ $\frac{D^{2}}{\lambda^{2}}+\frac{2(m+k) ! F_{\mu}^{2}(m+k)}{m !}+\frac{2(m+2 k) ! F_{\mu}^{2}(m+2 k)}{(m+k) !}$, the modification of which could be interpreted as an indicator of the nonlinearity $[40,41]$. For very small Lamb-Dicke nonlinearity values, the Rabi frequency has larger values for the larger values of the parameters $N$ and $D$. Therefore, we will discuss the results with larger values of the mean photon number $N$, and for two cases of the two trapped-ions interaction (without ion-ion interaction $D=0$ and with ion-ion interaction $D=10$ ). From the Hamiltonian of Equation (2), we find that the trapped-ion coupling $D$ plays an important role in generating the LQFI, LQU, and LGN non-classical correlations, where the interaction terms involving the two trappedions operators which naturally turns a superable state of the type $\left|1_{A} 0_{B}\right\rangle$ into a state: $\left(\alpha\left|1_{A} 0_{B}\right\rangle+\beta\left|0_{A} 1_{B}\right\rangle\right),|\alpha|^{2}+|\beta|^{2}=1$, which could be close to the maximally correlated state. Therefore, the considered trapped-ion coupling value $D=10$ is very acceptable to generating LQFI, LQU, and LGN non-classical correlations.

Figure 1a shows the dynamics of the time evolutions of the LQFI, LQU, and LGN non-classical correlations for the mean photon number $N=16$ with different values of the LD nonlinearity in the absence of the ID and the TIC effects. Firstly, the correlation functions are plotted when the vibrational mode state is initially in the coherent state. For a small value of the LD nonlinearity $\mu=0.08$, the local quantum Fisher information, the LQU, as well as the logarithmic negativity show that the state of the two trapped-ion qubits have different non-classical correlations. Note that the LQFI and LQU evolve approximately with the same irregular dynamical behavior. The LQFI amplitudes are always greater than those of the LQU. This agrees with the fact that the skew information is basically a special case of the quantum Fisher [25]. The generated strong non-classical correlations between the two trapped-ion qubits is due to the fact that In the Lamb-Dicke limit $\mu \ll 1$, an effective interaction causes a direct transition between the upper and the lower states of the two trapped-ion quibits without changing the state of the vibrational modes. This leads to the generation of the two trapped-ion entangled states. The dash-dot curve in Figure 1a shows that, after a short time (time-interval delay birth (TIDB) of entanglement), the logarithmic negativity arises suddenly and grows to show a partial entanglement. Then it vanishes suddenly. These processes indicate the sudden death-birth in the two trapped-ion entanglement dynamics [61,62]. The disentanglement appears during different time intervals. Note that the trapped-ion qubits have LQFI and LQU correlations during disentanglement time intervals. For the particular time-intervals $\lambda t \in[\pi, 3 \pi]$, the LQFI correlation is more stable than those of the LQU and the LGN. 
Figure $1 b, c$ shows the dependence of the created NCCs between the trapped-ion qubits on the LD nonlinearity. We find that the amplitudes and the frequencies of the logarithmic negativity are remarkably reduced, while the LQFI and LQU correlations between the trapped-ion qubits are enhanced. The time delay birth (TIDB) of the entanglement and the time to attain the maxima of the LQFI and LQU correlations are enhanced with the increase of the LD nonlinearity parameters. The magnitudes and robustness of the generated LQFI and LQU correlations against the high LD nonlinearity are larger than that of the logarithmic negativity.

Figure 2 displays the effect of the initial nonclassicality on the generated LQFI, LQU, and LGN non-classical correlation dynamics when the center-of-mass vibrational mode is initially in the even superposition coherent state. We note that, for a high initial mode nonclassicality, the LQFI, LQU, and LGN non-classical correlation dynamics can be accelerated and enhanced, see Figure 2a. The stability intervals of the LQFI are reduced.

For a high LD nonlinearity value, the LQFI, LQU, and LGN dynamics are shown in Figure $2 b$, with remarkable changes with respect to Figures $1 c$ and $2 a$. We find that the increase of both the LD nonlinearity and the initial mode nonclassicality reduce the two trapped-ion entanglement. We can observe the phenomenon of the sudden death-birth of the trapped-ion entanglement, as well as the disentanglement time intervals, while the LQFI and the LQU dynamics attain, respectively, quasi-regular oscillatory behaviors. Note that the phenomenon of sudden changes occurs in the dynamics of the local quantum Fisher information and the local quantum uncertainty. This phenomenon was predicted in several systems [63,64] and observed experimentally [65]. This phenomenon can be detected clearly in Figures 4 and 5. By comparing Figure 2 b with Figure 1c, we find that, when the vibrational state is initially in the even superposition coherent states, the increase of the initial nonclassicality leads to (1) the resulting LQFI and LQU correlations between trapped-ion qubits are accelerated and amplified, and they reach their peaks early, and (2) the sudden birth and death phenomena interval increases.

Figure 3 shows the dynamics of the correlation functions between the trapped-ion qubits under the intrinsic decoherence. By comparing Figures $1 a$ and $3 a$, we find that after a short time and small value of the LD nonlinearity $\mu=0.08$, the increase of the decoherence parameter $\gamma=0.01 \lambda$ leads to (1) roughly diminishing fluctuations in the LQFI, LQU, and LGN correlations, and (2) establishing steady correlations between the two trapped-ion qubits. The generated stationary correlations, induced by the decoherence, can be used for realizing quantum information processing. Figure $3 \mathrm{~b}$ shows the robustness of the generated two-trapped-ion correlations against the decoherence with high LD nonlinearity. From Figures $1 \mathrm{c}$ and $3 \mathrm{~b}$, we find that the influence of the decoherence parameter on the created LQFI and LQU correlations (owing to the unitary qubit-mode interactions) is weaker for the high LD nonlinearity $\mu=0.99$, and the generated stationary correlations do not emerge.

The dependence of the LQFI, LQU, and LGN correlation dynamics on the trapped-ion coupling (TIC) is shown in Figure 4 for $D=10 \lambda$. We find that the increase of the TIC parameter enhances the LQFI, LQU, and LGN correlations with more fluctuations. From Equations (8) and (9), we can interpret these increases and fluctuations due to the nonlinear terms of the time-dependent two trapped-ion density matrix elements depending explicitly on the trapped-ion coupling parameter $D \neq 0$. From Figure $4 a$, we observe that the LQFI, LQU, and LGN correlations have approximately the same irregular dynamical behavior with more fluctuations. The time-interval delay birth of the LGN entanglement disappears, and all the generated NCCs can be accelerated due to the coupling between the two trapped-ion qubits. The sudden death-birth intervals of the LGN entanglement vanish. The stability intervals of the LQFI, LQU, and LGN correlations can be enhanced in the Lamb-Dicke limit $\mu \ll 1$. Figure $4 \mathrm{~b}$ confirms the high initial even superposition nonclassicality effects on the LQFI, LQU, and LGN dynamics. The magnitudes and the frequencies of the correlation quantifiers are enhanced and accelerated.

Figure 5 illustrates the robustness of the generated LQFI, LQU, and LGN correlation, due to the ion-mode and the ion-ion interactions in the LD limit $\mu \ll 1$ for the two trapped- 
ion coupling $D=10 \lambda$ and the decoherence $\gamma=0.01 \lambda$. When the vibrational mode state is initially in the coherent state in Figure $5 \mathrm{a}$ and the even coherent state in Figure 5b. For this initial coherent state, the LQFI, LQU, and LGN quantifiers grow and reach their stationary correlations quicker than the ones of the even coherent state. By comparing Figure 5a with Figure $3 a$, the increase of the coupling between the two trapped-ions, $D=10 \lambda$, enhances and protects the generated stationary LQFI, LQU, and LGN correlations (which are induced by the decoherence).

Figure 6 displays the generated LQFI, LQU, and LGN correlations for high LD nonlinearity $\mu=0.99$ and the trapped-ion coupling $D=10 \lambda$. In this case, we can get a strong correlation after a long time. By comparing Figure 6a with Figure 1c (where $\lambda t \in[0,8 \pi]$ ), we find that, in the case where the high values of the LD nonlinearity and the trappedion coupling, the LQFI, LQU, and LGN quantifiers grow monotonically. In the case of Figure 6, with the increase of the LD nonlinearity and the trapped-ion coupling parameters, the generated two trapped-ion LGN entanglement is always larger than the LQFI and LQU correlations. The generated LQFI, LQU, and LGN correlations are more robust against decoherence.

\section{Conclusions}

In this paper, we have explored the dynamics of different kinds of two-trapped-ion correlations under intrinsic decoherence via local quantum Fisher information, the local quantum uncertainty, and the logarithmic negativity. The system engineering is designed such that the two-trapped-ions work as two diploe-coupled qubits in a Lamb-Dicke regime. In this study, we have focused on the cases where the center-of-mass vibrational modes are initially in coherent and even-superposition coherent states. The two-trapped-ion correlations have been analyzed under the effects of the Lamb-Dicke nonlinearity, the initial center-of-mass vibrational mode nonclassicality, as well as the trapped-ion coupling and the intrinsic decoherence. It is found that the LQFI and LQU evolve according to the fact that the skew information is a special case of the quantum Fisher information. The phenomenon of the sudden death-birth in the trapped-ion entanglement dynamics, as well as disentanglement time intervals, are observed and discussed for different physical parameters. For the high initial mode nonclassicality, the LQFI, LQU, and LGN nonclassical correlation can be accelerated and enhanced. The phenomenon of sudden changes that occurs in the local quantum Fisher information and the local quantum uncertainty can be controlled by the initial mode nonclassicality, the trapped-ion coupling, and the decoherence. Furthermore, the time delay birth of the entanglement and the time to reach the maxima of the LQFI and LQU correlations depend on the system parameters. The decoherence leads to stationary correlations, which depend on the LD nonlinearity, the initial mode nonclassicality, and the trapped-ion coupling. These generated stationary correlations can be useful for realizing quantum information processing.

Author Contributions: Conceptualization, A.-B.A.M.; Data curation, A.F., M.F.Y. and H.E.; Investigation, A.-B.A.M. and A.F.; Methodology, M.F.Y.; Supervision, H.E.; Visualization, A.-B.A.M. and A.F.; Writing original draft, A.-B.A.M. and H.E.; Writing review and editing. All authors have read and agreed to the published version of the manuscript.

Funding: This research received no external funding.

Institutional Review Board Statement: Not applicable.

Informed Consent Statement: Not applicable.

Data Availability Statement: Not applicable.

Conflicts of Interest: The authors declare no conflict of interest.

\section{References}

1. Nielsen, M.A.; Chuang, I.L. Quantum Computation and Quantum Information; Cambridge University Press: Cambridge, UK, 2000.

2. Jozsa, R.; Linden, N. On the role of entanglement in quantum-computational speed-up. Proc. R. Soc. A 2003, 459, 2011. [CrossRef] 
3. Lanyon, B.; Barbieri, M.; Almeida, M.; White, A. Experimental Quantum Computing without Entanglement. Phys. Rev. Lett. 2008, 101, 200501. [CrossRef] [PubMed]

4. Datta, A.; Vidal, G. Role of entanglement and correlations in mixed-state quantum computation. Phys. Rev. A 2007, 75, 042310. [CrossRef]

5. Bennett, C.H.; Bernstein, H.J.; Popescu, S.; Schumacher, B. Concentrating partial entanglement by local operations. Phys. Rev. A 1996, 53, 2046. [CrossRef]

6. Vidal, G.; Werner, R.F. Computable measure of entanglement. Phys. Rev. A 2002, 65, 032314. [CrossRef]

7. Wootters, W.K. Entanglement of Formation of an Arbitrary State of Two Qubits. Phys. Rev. Lett. 1998, 80, 2245. [CrossRef]

8. Melkikh, A.V. Quantum system: Wave function, entanglement and the uncertainty principle. Mod. Phys. Lett. B 2021, 35, 2150222. [CrossRef]

9. Ollivier, H.; Zurek, W.H. Quantum Discord: A Measure of the Quantumness of Correlations. Phys. Rev. Lett. 2001, 88, 017901. [CrossRef]

10. Mohamed, A.-B.A.; Eleuch, H. Quantum correlation control for two semiconductor microcavities connected by an optical fiber. Phys. Scr. 2017, 92, 065101. [CrossRef]

11. Virzi, S.; Rebufello, E.; Avella, A.; Piacentini, F.; Gramegna, M.; Berchera, I.R.; Degiovanni, I.P.; Genovese, M. Optimal estimation of entanglement and discord in two-qubit states. Sci. Rep. 2019, 9, 3030. [CrossRef]

12. Mohamed, A.-B.A.; Eleuch, H.; Ooi, C.H.R. Non-locality Correlation in Two Driven Qubits Inside an Open Coherent Cavity: Trace Norm Distance and Maximum Bell Function. Sci. Rep. 2019, 9, 19632. [CrossRef]

13. Abdel-Aty, A.-H.; Kadry, H.; Mohamed, A.-B.A.; Eleuch, H. Correlation dynamics of nitrogen vacancy centers located in crystal cavities. Sci. Rep. 2020, 10, 16640. [CrossRef]

14. A Obada, A.-S.F.; Mohamed, A.-B.A. Quantum correlations of two non-interacting ion's internal electronic states with intrinsic decoherence. Opt. Commun. 2013, 309, 236. [CrossRef]

15. Wigner, E.P.; Yanase, M.M. Information Contents Of Distributions. Proc. Natl. Acad. Sci. USA 1963, 49, 910. [CrossRef]

16. Wu, S.-X.; Zhang, J.; Yu, C.-S.; Song, H.-S. Uncertainty-induced quantum nonlocality. Phys. Lett. A 2014, 378, 344. [CrossRef]

17. Toóth, G. Multipartite entanglement and high-precision metrology. Phys. Rev. A 2012, 85, 022322. [CrossRef]

18. Girolami, D.; Souza, A.M.; Giovannetti, V.; Tufarelli, T.; Filgueiras, J.G.; Sarthour, R.S.; Soares-Pinto, D.O.; Oliveira, I.S.; Adesso, G. Quantum Discord Determines the Interferometric Power of Quantum States. Phys. Rev. Lett. 2014, 112, 210401. [CrossRef]

19. Hu, M.-L.; Hu, X.; Wang, J.; Peng, Y.; Zhang, Y.-R.; Fan, H. Quantum coherence and geometric quantum discord. Phys. Rep. 2018, 762, 1. [CrossRef]

20. Taddei, M.M.; Escher, B.M.; Davidovich, L.; de Matos Filho, R.L. Quantum Speed Limit for Physical Processes. Phys. Rev. Lett. 2013, 110, 050402. [CrossRef]

21. Sun, Z.; Ma, J.; Lu, X.-M.; Wang, X. Fisher information in a quantum-critical environment. Phys. Rev. A 2010, 82, 022306. [CrossRef]

22. Chapeau-Blondeau, F. Entanglement-assisted quantum parameter estimation from a noisy qubit pair: A Fisher information analysis. Phys. Lett. A 2017, 381, 1369. [CrossRef]

23. Dhar, H.S.; Bera, M.N.; Adesso, G. Characterizing non-Markovianity via quantum interferometric power. Phys. Rev. A 2015, 991, 032115. [CrossRef]

24. Slaoui, A.; Bakmou, L.; Daoud, M.; AhlLaamara, R. A comparative study of local quantum Fisher information and local quantum uncertainty in Heisenberg XY model. Phys. Lett. A 2019, 383, 2241. [CrossRef]

25. Luo, S. Wigner-Yanase Skew Information and Uncertainty Relations. Phys. Rev. Lett. 2003, 91, 180403. [CrossRef]

26. Gu, X.; Kockum, A.F.; Miranowicz, A.; Liu, Y.-X.; Nori, F. Microwave photonics with superconducting quantum circuits. Phys. Rep. 2017, 718-719, 1. [CrossRef]

27. Obada, A.-S.F.; Hessian, H.A.; Mohamed, A.-B.A.; Homid, A.H. Efficient protocol of N-bit discrete quantum Fourier transform via transmon qubits coupled to a resonator. Quantum Inf. Process. 2014, 13, 475. [CrossRef]

28. Denchev, V.S.; Boixo, S.; Isakov, S.V.; Ding, N.; Babbush, R.; Smelyanskiy, V.; Martinis, J.; Neven, H. What is the Computational Value of Finite-Range Tunneling? Phys. Rev. X 2016, 6, 031015. [CrossRef]

29. Georgescu, I.M.; Ashhab, S.; Nori, F. Quantum simulation. Rev. Mod. Phys. 2014, 86, 153. [CrossRef]

30. Häffner, H.; Roos, C.F.; Blatt, R. Quantum computing with trapped ions. Phys. Rep. 2008, 469, 155. [CrossRef]

31. Brown, K.R.; Chiaverini, J.; Sage, J.M.; Häffner, H. Materials challenges for trapped-ion quantum computers. Nat. Rev. Mater. 2021. [CrossRef]

32. Cirac, J.I.; Zoller, P. Quantum Computations with Cold Trapped Ions. Phys. Rev. Lett. 1995, 74, 4091. [CrossRef]

33. van Mourik, M.W.; Martinez, E.A.; Gerster, L.; Hrmo, P.; Monz, T.; Schindler, P.; Blatt, R. Coherent rotations of qubits within a surface ion-trap quantum computer. Phys. Rev. A 2020, 102, 022611. [CrossRef]

34. Wei, L.F.; Nori, F. New exclusion limits on dark gauge forces from proton Bremsstrahlung in beam-dump data. Phys. Lett. A 2003, 320, 131. [CrossRef]

35. Li, L.-X.; Guo, G.-C. Quantum logic gate operation between different ions in a trap. Phys. Rev. A 1999, 60, 696. [CrossRef]

36. Blockley, C.A.; Walls, D.F. Cooling of a trapped ion in the strong-sideband regime. Phys. Rev. A 1993, 47, 2115. [CrossRef]

37. Cirac, J.I.; Blatt, R.; Parkins, A.S.; Zoller, P. Quantum collapse and revival in the motion of a single trapped ion. Phys. Rev. A 1994, 49, 1202. [CrossRef] 
38. Jaynes, E.T.; Cummings, F.W. Comparison of quantum and semiclassical radiation theories with application to the beam maser. Proc. IEEE 1963, 51, 89. [CrossRef]

39. Blockey, C.A.; Walls, D.F.; Risken, H. Quantum Collapses and Revivals in a Quantized Trap. Europhys. Lett. 1992, 17, 509. [CrossRef]

40. Vogel, W.; de Matos Filho, R.L. Nonlinear Jaynes-Cummings dynamics of a trapped ion. Phys. Rev. A 1995, 52, 4214. [CrossRef]

41. Krumm, F.; Vogel, W. Time-dependent nonlinear Jaynes-Cummings dynamics of a trapped ion. Phys. Rev. A 2018, 97, 043806. [CrossRef]

42. Cheng, X.-H.; Arrazola, I.; Pedernales, J.S.; Lamata, L.; Chen, X.; Solano, E. Nonlinear quantum Rabi model in trapped ions. Phys. Rev. A 2018, 97, 023624. [CrossRef]

43. Zhang, S.; Zhang, J.-Q.; Wu, W.; Bao, W.-S.; Guo, C. Fast cooling of trapped ion in strong sideband coupling regime. New J. Phys. 2021, 23,023018. [CrossRef]

44. Hessian, H.A.; Mohamed, A.-B.A. Quasi-Probability Distribution Functions for a Single Trapped Ion Interacting with a Mixed Laser Field. Laser Phys. 2008, 18, 1217. [CrossRef]

45. Wei, L.F.; Liu, Y.-X.; Nori, F. Engineering quantum pure states of a trapped cold ion beyond the Lamb-Dicke limit. Phys. Rev. A 2004, 70, 063801. [CrossRef]

46. Simeonov, L.S.; Vitanov, N.V.; Ivanov, P.A. Compensation of the trap-induced quadrupole interaction in trapped Rydberg ions. Sci. Rep. 2019, 9, 7340. [CrossRef]

47. Harlander, M.; Lechner, R.; Brownnutt, M.; Blatt, R.; Hänsel, W. Trapped-ion antennae for the transmission of quantum information. Nature 2011, 471, 200. [CrossRef]

48. Li, W.; Lesanovsky, I. Entangling quantum gate in trapped ions via Rydberg blockade. App. Phys. B 2014, 114, 37. [CrossRef]

49. Zhang, J.Q.; Xiong, W.; Zhang, S.; Li, Y.; Feng, M. Generating the Schrodinger cat state in a nanomechanical resonator coupled to a charge qubit. Ann. Phys. 2015, 527, 180. [CrossRef]

50. Mohamed, A.-B.A.; Hashem, M.; Eleuch, H. Enhancing the Generated Stable Correlation in a Dissipative System of Two Coupled Qubits inside a Coherent Cavity via Their Dipole-Dipole Interplay. Entropy 2019, 21, 672. [CrossRef]

51. Sharma, S.S.; Sharma, N.K. Intrinsic decoherence effects on tripartite GHZ state generation using a trapped ion coupled to an optical cavity. J. Opt. B Quantum Semiclass. Opt. 2005, 7, 230. [CrossRef]

52. Milburn, G.J. Intrinsic decoherence in quantum mechanics. Phys. Rev. A 1991, 44, 5401. [CrossRef] [PubMed]

53. Retzker, A.; Solano, E.; Reznik, B. Tavis-Cummings model and collective multiqubit entanglement in trapped ions. Phys. Rev. A 2007, 75, 022312. [CrossRef]

54. Mohamed, A.-B.A.; Hessian H.A.; Obada A.-S.F. Nonclassical effects in a nonlinear two trapped-particles system under intrinsic decoherence. Chaos Solitons Fractals 2021, 146, 110857. [CrossRef]

55. Solano, E.; Milman, P.; Filho, R.L.d.; Zagury, N. Manipulating motional states by selective vibronic interaction in two trapped ions. Phys. Rev. A 2000, 62, 021401. [CrossRef]

56. Mohamed, A.-B.A.; Eleuch, H.; Raymond Ooi, C.H. Quantum coherence and entanglement partitions for two driven quantum dots inside a coherent micro cavity. Phys. Lett. A 2019, 383, 125905. [CrossRef]

57. Rai, A.; Das, S.; Agarwal, G.S. Quantum entanglement in coupled lossy waveguides. Opt. Express 2010, 18, 6241. [CrossRef]

58. Girolami, D.; Tufarelli, T.; Adesso, G. Characterizing Nonclassical Correlations via Local Quantum Uncertainty. Phys. Rev. Lett. 2013, 110, 240402. [CrossRef]

59. Mohamed, A.-B.A. Quantum correlation of correlated two qubits interacting with a thermal field. Phys. Scr. 2012, 85, 055013. [CrossRef]

60. Mohamed, A.-B.A.; Metwally, N. Non-classical correlations based on skew information for an entangled two qubit-system with non-mutual interaction under intrinsic decoherence. Ann. Phys. 2017, 381, 137. [CrossRef]

61. Yu, T.; Eberly, J.H. Sudden Death of Entanglement. Science 2009, 323, 598. [CrossRef]

62. Mohamed, A.-B.A. Bipartite non-classical correlations for a lossy two connected qubit-cavity systems: Trace distance discord and Bell's non-locality. Quantum Inf. Process 2018, 17, 96. [CrossRef]

63. Mohamed, A.-B.A.; Eleuch, H. Generation and robustness of bipartite non-classical correlations in two nonlinear microcavities coupled by an optical fiber. J. Opt. Soc. Am. B 2018, 35, 47. [CrossRef]

64. Mohamed, A.-B.A.; Farouk, A.; Yassen, M.F.; Eleuch, H. Quantum Correlation via Skew Information and Bell Function Beyond Entanglement in a Two-Qubit Heisenberg XYZ Model: Effect of the Phase Damping. Appl. Sci. 2020, 10, 3782. [CrossRef]

65. Xu, J.-S.; Xu, X.-Y.; Li, C.-F.; Zhang, C.-J.; Zou, X.-B.; Guo, G.-C. Experimental investigation of classical and quantum correlations under decoherence. Nat. Commun. 2010, 1, 7. [CrossRef] 